\title{
Some sufficient conditions for the absolute Cesàro summability of series
}

\author{
By J. M. HysLop.
}

(Received 25th January, 1939. Read 3rd February, 1939.)

1. Let $c_{n}^{(k)}$ denote the $n$-th Cesàro mean of order $k$ for the series $\sum a_{n}$, that is,

where

$$
c_{n}^{(k)}=S_{n}^{(k)} \mid \mathcal{A}_{n}^{(k)}
$$

and let

$$
A_{n}^{(k)}=\left(\begin{array}{c}
k+n \\
n
\end{array}\right), S_{n}^{(k)}=\sum_{\nu=0}^{n}\left(\begin{array}{c}
k+n-v \\
n-\nu
\end{array}\right) a_{\nu}
$$

$$
\begin{aligned}
& a_{n}^{(k)}=c_{n}^{(k)}-c_{n-1}^{(k)}, n \geqq 1, \\
& a_{0}^{(k)}=c_{0}^{(k)} .
\end{aligned}
$$

Then the series $\Sigma a_{n}$ is said to be summable $(C, k)$ to the sum $s$ if $\mathbf{c}_{n}^{(k)} \rightarrow s$ as $n \rightarrow \infty$, and absolutely summable $(C, k)$, or summable $\mid C, k\}$, if $\Sigma\left|a_{n}^{(k)}\right|$ is convergent. Throughout this paper we shall suppose that $k \geqq 0$.

It is well known that a series which is summable or absolutely summable $(C, k)$ is respectively summable or absolutely summable $(C, \kappa)$ for every $\kappa>k$.

An important relation connecting $a_{n}^{(k)}$ and $a_{n}$ is provided by the formal identity ${ }^{1}$

$$
\sum_{n=0}^{\infty} n A_{n}^{(k)} a_{n}^{(k)} x^{n}=(1-x)^{-k} \sum_{n=0}^{\infty} n a_{n} x^{n}
$$

2. The object of this note is to obtain some simple relations connecting Cesàro and absolute Cesàro summability. It is well known and, in any case, follows at once from the definition of absolute summability that, if a series is summable $|C, k|$, it is also summable $(C, k)$. The converse of course is not true. Indeed an example has

1 E. Kngbetliantz, Bull. des Scionces Math. (2), 49 (1925), 234-256. 
been constructed ${ }^{1}$ to show that a convergent series need not be absolutely summable by Abel's method and, a fortiori $i^{2}$, need not be summable $|C, k|$ for any value of $k$. In view of this result the inference

$$
\Sigma a_{n}=s(C, k) \text { implies } \Sigma a_{n}=s|C, \kappa| \text { for some } \kappa
$$

is bound to be false, and it is natural to ask what alteration in the hypothesis would suffice to make the above conclusion valid. An answer is suggested by a recent paper ${ }^{3}$ dealing with series which are more than summable $(C, k)$ in the sense that they satisfy a condition of the form

$$
c_{n}^{(k)}=s+O\left(n^{-\delta}\right), 0<\delta \leqq 1 .
$$

It will be shown that such series and series satisfying a slightly wider condition are always summable $|C, \kappa|$ for some $\kappa$.

3. We proceed to obtain these results.

THEOREM 1. If the series $\Sigma a_{n}$ satisfies the relation

$$
c_{n}^{(k)}=s+\phi_{n}, \phi_{n} \rightarrow 0
$$

where $\Sigma n^{-1}\left|\phi_{n}\right|$ is convergent, then $\Sigma a_{n}$ is summable $|C, \kappa|$ for $\kappa \geqq k+1$.

Without loss of generality we may suppose that $k=z+1$.

From (1) we have

so that

$$
\begin{aligned}
\sum_{n=0}^{\infty} n A_{n}^{(k+1)} a_{n}^{(k+1)} x^{n} & =(1-x)^{-k-1} \sum_{n=0}^{\infty} n a_{n} x^{n} \\
& =(1-x)^{-1} \sum_{n=0}^{\infty} n A_{n}^{(k)} a_{n}^{(k)} x^{n},
\end{aligned}
$$

$$
\begin{aligned}
n A_{n}^{(k+1)} a_{n}^{(k+1)} & =\sum_{\nu=0}^{n} \nu A_{\nu}^{(k)} a_{\nu}^{(k)} \\
& =n A_{n}^{(k)} c_{n}^{(k)}-\sum_{\nu=0}^{n-1} c_{\nu}^{(k)}\left\{(\nu+1) A_{\nu+1}^{(k)}-\nu A_{\nu}^{(k)}\right\} \\
& =n A_{n}^{(k)} \phi_{n}-\sum_{\nu=0}^{n-1} \phi_{\nu}\left\{(\nu+1) A_{\nu+1}^{(k)}-\nu A_{\nu}^{(k)}\right\} \\
& =n A_{n}^{(k)} \phi_{n}-(k+1) \sum_{\nu=0}^{n-1} \phi_{\nu} A_{\nu}^{(k)} \\
& =T_{1}-T_{2},
\end{aligned}
$$

1 J. M. Whittaker, Proc. Edinburgh Math. Soc. (2), 2 (1930), 1-5.

${ }^{2}$ M. Fekete, Proc. Edinburgh Math. Soc. (2), 3 (1933), 132-134.

3. M. Hyslop, Proc. Edinburgh Math. Soc. (2), 5 (1938), 182-201. 
say. Since $A_{n}^{(k)} \sim n^{k} / \Gamma(k+1)$ it is sufficient to prove that $\Sigma n^{-k-2}\left|T_{1}\right|$ and $\sum n^{-k-2}\left|T_{2}\right|$ are convergent. The first of these follows at once from the hypothesis. Also

so that

$$
T_{2}=(k+1) \sum_{\nu=1}^{n} \phi_{\nu} A_{\nu}^{(k)}+o\left(n^{k}\right)
$$

$$
\begin{aligned}
\sum_{n=1}^{\infty} n^{-k-2}\left|T_{2}\right| & <A\left\{\sum_{\nu=1}^{\infty}\left|\phi_{\nu}\right| v^{k} \sum_{n=\nu}^{\infty} n^{-k-2}+\sum_{n=1}^{\infty} n^{-2}\right\} \\
& <A\left\{\sum_{\nu=1}^{\infty} \nu^{-1}\left|\phi_{\nu}\right|+\sum_{n=1}^{\infty} n^{-2}\right\} \\
& <\infty
\end{aligned}
$$

and the theorem follows.

The particular case of this theorem when $\phi_{n}=O\left(n^{-\delta}\right), 0<\delta \leqq 1$, deserves special consideration. We have just proved that series which satisfy the condition

$$
c_{n}^{(k)}=s+O\left(n^{-\delta}\right), 0<\delta \leqq 1
$$

are summable $|C, k+1|$, but it is possible to obtain more precise information about such series.

THeORem 2. If the series $\sum a_{n}$ satisfies condition (3) it is summable $|C, \kappa|$ for $\kappa>k+1-\delta$.

Without loss of generality we may suppose that

$$
k+1-\delta<k<k+1 \text {. }
$$

From (1) we obtain

$$
\begin{aligned}
n A_{n}^{(\kappa)} a_{n}^{(\kappa)} & =\sum_{\nu=0}^{n} \nu A_{\nu}^{(k)} a_{\nu}^{(k)} A_{n-\nu}^{(\kappa-k-1)} \\
& =n A_{n}^{(k)} c_{n}^{(k)}-\sum_{\nu=0}^{n-1} c_{\nu}^{(k)}\left\{(\nu+1) A_{\nu+1}^{(k)} A_{n-\nu-1}^{(\kappa-k-1)}-\nu A_{\nu}^{(k)} A_{n-\nu}^{(\kappa-k-1)}\right\} \\
& =n A_{n}^{(k)} \phi_{n}-\sum_{\nu \cdots 0}^{n-1} \phi_{\nu}\left\{(\nu+1) A_{\nu+1}^{(k)} A_{n-\nu-1}^{(\kappa-k-1)}-\nu A_{\nu}^{(k)} A_{n-\nu}^{(\kappa-k-1)}\right\} \\
& =T_{1}-T_{2},
\end{aligned}
$$

say. Now

$$
\begin{aligned}
\sum n^{-\kappa-1} T_{1} \mid & <A \sum n^{-\kappa-1} n^{k+1}\left|\phi_{n}\right| \\
& <A \sum n^{k-\kappa-\delta}<\infty
\end{aligned}
$$

and we have to show that the same is true of $T_{2}$. 
The difference involved in the $T_{2}$ summation is equal to

$$
\begin{aligned}
& \frac{(k+1) \ldots(k+\nu+1)(\kappa-k) \ldots(\kappa-k+n-\nu-2)}{\nu !(n-\nu-1) !}- \\
& (k+1) \ldots(k+\nu)(\kappa-k) \ldots(\kappa-k+n-\nu-1) \\
(\nu-1) !(n-\nu) ! & \frac{(k+1) \ldots(k+\nu)(\kappa-k) \ldots(\kappa-k+n-\nu-2)}{\nu !(n-\nu) !} \times \\
& \{(k+\nu+1)(n-v)-\nu(\kappa-k+n-\nu-1)\} \\
= & \frac{(k+1) \ldots(k+\nu)(\kappa-k) \ldots(\kappa-k+n-\nu-2)}{\nu !(n-\nu) !} \times \\
& \{(k+1)(n-\nu)-\nu(\kappa-k-1)\} \\
= & (k+1) A_{\nu}^{(k)} A_{n-\nu-1}^{(\kappa-k-1)}-\nu A_{\nu}^{(k)} A_{n-\nu}^{(\kappa-k-2)},
\end{aligned}
$$

and we therefore have $T_{2}=T_{2,1}-T_{2,2}$, where

$$
\begin{aligned}
& T_{2,1}=(k+1) \sum_{\nu=0}^{n-1} \phi_{\nu} A_{\nu}^{(k)} A_{n-1-\nu}^{(\kappa-k-1)}, \\
& T_{2,2}=\sum_{\nu=0}^{n-1} \nu \phi_{\nu} A_{\nu}^{(k)} A_{n-\nu}^{(\kappa-k-2)} .
\end{aligned}
$$

Now

$$
\begin{aligned}
T_{2,2} & =O\left\{n^{k+1-\delta} \sum_{\nu=0}^{n-1} A_{n-\nu}^{(\kappa-k-2)} \mid\right\} \\
& =O\left\{n^{k+1-\delta} \sum_{\nu=0}^{\infty}(\nu+1)^{\kappa-k-2}\right\} \\
& =O\left(n^{k+1-\delta}\right),
\end{aligned}
$$

so that $\sum n^{-\kappa-1}\left|T_{2,2}\right|$ is convergent.

Also

$$
\begin{aligned}
T_{2,1} & =O\left\{\sum_{\nu=0}^{n-1} A_{\nu}^{(k-\delta)} A_{n-1-\nu}^{(\kappa-k-1)}\right\} \\
& =O\left\{A_{n-1}^{(\kappa-\delta)}\right\}=O\left(n^{\kappa-\delta}\right),
\end{aligned}
$$

except in the case when $k=0, \delta=1$. In this case

$$
\begin{aligned}
T_{2,1} & =O\left\{\sum_{\nu=1}^{\frac{1}{2} n} \nu^{-1} A_{n-1-\nu}^{(\kappa-1) !}\right\}+O\left\{\sum_{\frac{1}{2} n}^{n-1} \nu^{-1} A_{n-1-\nu}^{(\kappa-1)}\right\}+A_{0}^{(-1)} A_{n-1}^{(\kappa-1)} \\
& =O\left\{n^{\kappa-1} \sum_{\nu=1}^{\frac{1}{2} n} \nu^{-1}\right\}+O\left\{n^{-1} \sum_{\nu=0}^{n-1} A_{n-1-\nu}^{(\kappa-1)}\right\}+O\left\{n^{\kappa-1}\right\} \\
& =O\left(n^{\kappa-1} \log n\right)+O\left(n^{\kappa-1}\right) \\
& =O\left(n^{\kappa-1} \log n\right) .
\end{aligned}
$$

Hence $\Sigma n^{-\kappa-1}\left|T_{2,1}\right|$ is convergent and the theorem is proved. 
It is not difficult to see that the inequality in the conclusion of Theorem 2 cannot be replaced by the corresponding equality for, in the case of the series $1-1+1 \ldots$,

$$
c_{n}^{(1)}=\frac{1}{2}+O\left(\frac{1}{n}\right)
$$

and the series is not summable $|C, 1|$. The example $\Sigma(-1)^{n} n^{-1}$ illustrates the same point for the case $k=0$. A simple example illustrating the use of Theorem 1 is provided by the series $\Sigma(-1)^{n}(\log n)^{-a}$, where $a>1$.

4. It has been shown elsewhere that if the series $\Sigma n^{\delta} a_{n}$, $0<\delta \leqq 1$, is bounded $(C, k)$, where $k$ is zero or a positive integer, then the series $\sum a_{n}$ satisfies condition (3). We therefore have the following theorem.

Theorem 3. If the series $\Sigma n^{\delta} a_{n}, 0<\delta \leqq 1$, is bounded $(C, k)$, where $k$ is zero or a positive integer, then the series $\Sigma a_{n}$ is summable $|C, \kappa|$ for $\kappa>k+1-\delta$.

The restriction that $k$ is to be a positive integer is probably quite unnecessary, but the proof of the theorem for general positive $k$ would almost certainly be cumbered by much heavy algebraical work. It is easy to show, however, that, in the particular case $\delta=1$, the theorem is true for all values of $k \geqq 0$. Indeed it has been pointed out to me by a Referee that the theorem is true when $k$ is of the form $i+\rho$, where $i$ is zero or a positive integer and $0<\rho<\delta$. His proof is as follows. Choose $\delta^{\prime}$ such that $\rho<\delta^{\prime}<\delta$. Then, by a well-known theorem ${ }^{2}$ on Cesàro summability, the series $\sum n^{\delta^{\prime}-\rho} a_{n}$ is bounded $\left(C, i+\delta^{\prime}-\delta\right)$ and therefore bounded $(C, i)$. Since $i$ is a positive integer it follows that $\Sigma a_{n}$ is summable $|C, \kappa|$ for

1 J. M. Hyslop, loc. cit., 194. 436.

2 Cf. G. H. Hardy and J. E. Littlewood, Proc. London Math. Soc. (2), 11 (1912), 
$\kappa>i+1+\rho-\delta^{\prime}$. Hence, since $\delta^{\prime}$ is arbitrary, $\Sigma a_{n}$ is summable $|C, \kappa|$ for $\kappa>k+1-\delta$.

All three theorems may be regarded as tests for absolute summability and, although it is the least general, the third is the most convenient in application.

The University,

Glasgow. 